\title{
ON ERROR FORMULAS FOR APPROXIMATION BY SUMS OF UNIVARIATE FUNCTIONS
}

\author{
VUGAR E. ISMAILOV
}

Received 8 February 2005; Revised 1 December 2005; Accepted 12 March 2006

The purpose of the paper is to develop a new method for obtaining explicit formulas for the error of approximation of bivariate functions by sums of univariate functions. It should be remarked that formulas of this type have been known only for functions defined on a rectangle with sides parallel to coordinate axes. Our method, based on a maximization process over closed bolts, allows the consideration of functions defined on a hexagon or octagon with sides parallel to coordinate axes.

Copyright (c) 2006 Hindawi Publishing Corporation. All rights reserved.

\section{Introduction}

It is well known that the approximation of functions of several variables by simple combinations of univariate functions is of both theoretical and practical significance. This type of approximation has arisen, for example, in connection with the classical functional equations [5], the numerical solution of certain elliptic PDE boundary value problems [4], and dimension theory [15].

Consider the above-mentioned approximation in its simplest form, that is, an approximation of a continuous function $f(x, y)$ defined on a compact set $Q \subset \mathbb{R}^{2}$ by functions from the manifold

$$
D=\{\varphi(x)+\psi(y)\}
$$

where $\varphi(x), \psi(y)$ are defined and continuous on the projections of $Q$ onto the coordinate axes $x$ and $y$, respectively. The error in this approximation is defined as the distance from $f$ to $D$ :

$$
E(f, Q)=\operatorname{dist}(f, D)=\inf _{(\varphi+\psi) \in D}\|f-\varphi-\psi\|_{C(Q)} .
$$

A function $\varphi_{0}(x)+\psi_{0}(y)$ from $D$, if it exists, is called an extremal element or a best approximation if

$$
E(f, Q)=\left\|f-\varphi_{0}-\psi_{0}\right\|
$$

Hindawi Publishing Corporation

International Journal of Mathematics and Mathematical Sciences

Volume 2006, Article ID 65620, Pages 1-11

DOI 10.1155/IJMMS/2006/65620 
In some applications, one may be interested in computational techniques for the error of approximation. Diliberto and Straus [6] were the first to establish a formula for $E(f, R)$, where $R$ is a rectangle with sides parallel to coordinate axes. Their formula contains the supremum over all closed lightning bolts (for this terminology, see $[1,8,9,11$, 13]). Later the same formula was established by other authors differently in rectangular case (see [13]) and for more general sets (see $[9,11])$. Although the formula is valid for all continuous functions, it is not easily calculable. Some authors have been seeking easily calculable formulas for the approximation error by considering not the whole space but some subsets of continuous functions. For example, Rivlin and Sibner [14] showed that for a function $f(x, y)$ with the continuous nonnegative derivative $\partial^{2} f / \partial x \partial y$ on a rectangle $R=\left[a_{1}, b_{1}\right] \times\left[a_{2}, b_{2}\right]$ the approximation error can be calculated using the formula

$$
E(f, R)=\frac{1}{4}\left[f\left(a_{1}, a_{2}\right)+f\left(b_{1}, b_{2}\right)-f\left(a_{1}, b_{2}\right)-f\left(b_{1}, a_{2}\right)\right] .
$$

Babaev [2] generalized this result and proved that the formula is valid for a continuous function $f(x, y)$ with the nonnegative difference $\Delta_{h_{1} h_{2}} f$. Formulas of this type for some special classes of bivariate functions were established by the author in [10].

It should be said that the known techniques for obtaining the formulas of this type considerably use a geometrical structure of the rectangle $R$, the fact that $R$ is a Cartesian product of two line segments.

Our purpose is to develop a new method for obtaining explicit formulas providing precise and easy computation of $E(f, Q)$ on some simple polygons $Q$ with sides parallel to coordinate axes. By simple polygons we mean polygons with the total number of vertices not more than 8 . This method is based on the herein developed bolts maximization process and can be used in alternative proofs of the known results from $[2,10,14]$. First, we show efficiency of the method in the example of a hexagon with sides parallel to coordinate axes. Then we formulate two theorems, which can be proved in a similar way, and touch some aspects of the question about the case of an arbitrary polygon with sides parallel to coordinate axes. The condition posed on sides of polygons is essential for our method. This has several reasons, which get clear through the proof of Theorem 2.1. Here we are able to explain one of these reasons: by [8, Theorem 3], a continuous function $f(x, y)$ defined on a polygon with sides parallel to coordinate axes has an extremal element, the existence of which is required by our method. Now, let $K$ be a rectangle (not speaking about polygons) with sides not parallel to coordinate axes. Does any function $f \in C(K)$ have an extremal element? No one knows (see [8]).

In the sequel, all the considered polygons are assumed to have sides parallel to coordinate axes.

\section{The maximization process}

Let $H$ be a closed hexagon. It is clear that $H$ can be uniquely represented in the form

$$
H=R_{1} \cup R_{2},
$$

where $R_{1}, R_{2}$ are rectangles and there does not exist any rectangle $R$ such that $R_{1} \subset R \subset H$ or $R_{2} \subset R \subset H$. 
A bolt of lightning (see [1]) in $H$ is a finite ordered subset $p=\left\{p_{1}, p_{2}, \ldots, p_{n}\right\}$ of $H$ such that $p_{i} \neq p_{i+1}$, each line segment $p_{i} p_{i+1}$ (unit of the bolt) is parallel to the coordinate axis $x$ or $y$, and two adjacent units $p_{i} p_{i+1}$ and $p_{i+1} p_{i+2}$ are perpendicular. A bolt of lightning $p$ is said to be closed if $p_{n} p_{1} \perp p_{1} p_{2}$ (in this case, $n$ is an even number). We associate each closed bolt $p=\left\{p_{1}, p_{2}, \ldots, p_{2 n}\right\}$ with the following functional:

$$
l(f, p)=\frac{1}{2 n} \sum_{k=1}^{2 n}(-1)^{k-1} f\left(p_{k}\right) .
$$

Denote by $M(H)$ the class of continuous functions $f(x, y) \in C(H)$ satisfying the condition

$$
f\left(x_{1}, y_{1}\right)+f\left(x_{2}, y_{2}\right)-f\left(x_{1}, y_{2}\right)-f\left(x_{2}, y_{1}\right) \geq 0
$$

for any rectangle $\left[x_{1}, x_{2}\right] \times\left[y_{1}, y_{2}\right] \subset H$.

Theorem 2.1. Let $H$ be a hexagon and let (2.1) be its representation. Let $f(x, y) \in M(H)$. Then

$$
E(f, H)=\max \left\{|l(f, h)|,\left|l\left(f, r_{1}\right)\right|,\left|l\left(f, r_{2}\right)\right|\right\},
$$

where $h, r_{1}, r_{2}$ are closed bolts formed by vertices of the polygons $H, R_{1}, R_{2}$, respectively.

Proof. Without loss of generality, we may assume that the rectangles $R_{1}$ and $R_{2}$ are of the following form:

$$
R_{1}=\left[a_{1}, a_{2}\right] \times\left[b_{1}, b_{3}\right], \quad R_{2}=\left[a_{1}, a_{3}\right] \times\left[b_{1}, b_{2}\right], \quad a_{1}<a_{2}<a_{3}, b_{1}<b_{2}<b_{3} .
$$

Introduce the notation

$$
\begin{gathered}
f_{11}=f\left(a_{1}, b_{1}\right), \quad f_{12}=-f\left(a_{1}, b_{2}\right), \quad f_{13}=-f\left(a_{1}, b_{3}\right) ; \\
f_{21}=-f\left(a_{2}, b_{1}\right), \quad f_{22}=-f\left(a_{2}, b_{2}\right), \quad f_{23}=f\left(a_{2}, b_{3}\right) ; \\
f_{31}=-f\left(a_{3}, b_{1}\right), \quad f_{32}=f\left(a_{3}, b_{2}\right) .
\end{gathered}
$$

It is clear that

$$
\begin{gathered}
\left|l\left(f, r_{1}\right)\right|=\frac{1}{4}\left(f_{11}+f_{13}+f_{23}+f_{21}\right), \\
\left|l\left(f, r_{2}\right)\right|=\frac{1}{4}\left(f_{11}+f_{12}+f_{32}+f_{31}\right), \\
|l(f, h)|=\frac{1}{6}\left(f_{11}+f_{13}+f_{23}+f_{22}+f_{32}+f_{31}\right) .
\end{gathered}
$$
ting

Let $p=\left\{p_{1}, p_{2}, \ldots, p_{2 n}\right\}$ be any closed bolt. We group the points $p_{1}, p_{2}, \ldots, p_{2 n}$ by put-

$$
p_{+}=\left\{p_{1}, p_{3}, \ldots, p_{2 n-1}\right\}, \quad p_{-}=\left\{p_{2}, p_{4}, \ldots, p_{2 n}\right\} .
$$

First, assume that $l(f, p) \geq 0$. We apply the following algorithm, hereafter called the maximization process over closed bolts, to the bolt $p$. 
Step 1. Consider sequentially units $p_{i} p_{i+1}\left(i=\overline{1,2 n}, p_{2 n+1}=p_{1}\right)$ with the vertices $p_{i}\left(x_{i}, y_{i}\right)$, $p_{i+1}\left(x_{i+1}, y_{i+1}\right)$ having equal abscissae: $x_{i}=x_{i+1}$. Then we have four cases.

(1) $p_{i} \in p_{+}$and $y_{i+1}>y_{i}$. In this case, replace the unit $p_{i} p_{i+1}$ by a new unit $q_{i} q_{i+1}$ with vertices $q_{i}=\left(a_{1}, y_{i}\right), q_{i+1}=\left(a_{1}, y_{i+1}\right)$.

(2) $p_{i} \in p_{+}$and $y_{i+1}<y_{i}$. In this case, replace the unit $p_{i} p_{i+1}$ by a new unit $q_{i} q_{i+1}$ with vertices $q_{i}=\left(a_{2}, y_{i}\right), q_{i+1}=\left(a_{2}, y_{i+1}\right)$ if $b_{2}<y_{i} \leq b_{3}$ or with vertices $q_{i}=\left(a_{3}, y_{i}\right)$, $q_{i+1}=\left(a_{3}, y_{i+1}\right)$ if $b_{1} \leq y_{i} \leq b_{2}$.

(3) $p_{i} \in p_{-}$and $y_{i+1}<y_{i}$. In this case, replace $p_{i} p_{i+1}$ by a new unit $q_{i} q_{i+1}$ with vertices $q_{i}=\left(a_{1}, y_{i}\right), q_{i+1}=\left(a_{1}, y_{i+1}\right)$.

(4) $p_{i} \in p_{-}$and $y_{i+1}>y_{i}$. In this case, replace $p_{i} p_{i+1}$ by a new unit $q_{i} q_{i+1}$ with vertices $q_{i}=\left(a_{2}, y_{i}\right), q_{i+1}=\left(a_{2}, y_{i+1}\right)$ if $b_{2}<y_{i+1} \leq b_{3}$ or with vertices $q_{i}=\left(a_{3}, y_{i}\right), q_{i+1}=$ $\left(a_{3}, y_{i+1}\right)$ if $b_{1} \leq y_{i+1} \leq b_{2}$.

Since $f \in M(H)$, it is not difficult to verify that

$$
\begin{aligned}
f\left(p_{i}\right)-f\left(p_{i+1}\right) & \leq f\left(q_{i}\right)-f\left(q_{i+1}\right) \quad \text { for cases (1) and (2), } \\
-f\left(p_{i}\right)+f\left(p_{i+1}\right) & \leq-f\left(q_{i}\right)+f\left(q_{i+1}\right) \quad \text { for cases (3) and (4). }
\end{aligned}
$$

It is clear that after Step 1 the bolt $p$ is replaced by the ordered set $q=\left\{q_{1}, q_{2}, \ldots, q_{2 n}\right\}$. We say not a bolt but an ordered set because of a possibility of coincidence of some successive points $q_{i}, q_{i+1}$ (this, e.g., may happen if the first case takes place for units $p_{i-1} p_{i}$ and $p_{i+1} p_{i+2}$ ). Exclude simultaneously successive and coincident points from $q$. Then we obtain some closed bolt, which we denote by $q^{\prime}=\left\{q_{1}^{\prime}, q_{2}^{\prime}, \ldots, q_{2 m}^{\prime}\right\}$. It is not difficult to understand that all points of the bolt $q^{\prime}$ are located on straight lines $x=a_{1}, x=a_{2}, x=a_{3}$.

From inequalities (2.9) and the fact that $2 m \leq 2 n$, we deduce that

$$
l(f, p) \leq l\left(f, q^{\prime}\right)
$$

Step 2. Consider sequentially units $q_{i}^{\prime} q_{i+1}^{\prime}\left(i=\overline{1,2 m}, q_{2 m+1}^{\prime}=q_{1}^{\prime}\right)$ with the vertices $q_{i}^{\prime}=$ $\left(x_{i}^{\prime}, y_{i}^{\prime}\right), q_{i+1}^{\prime}=\left(x_{i+1}^{\prime}, y_{i+1}^{\prime}\right)$ having equal ordinates: $y_{i}^{\prime}=y_{i+1}^{\prime}$. Then we have four cases.

(1) $q_{i}^{\prime} \in q_{+}^{\prime}$ and $x_{i+1}^{\prime}>x_{i}^{\prime}$. In this case, replace the unit $q_{i}^{\prime} q_{i+1}^{\prime}$ by a new unit $p_{i}^{\prime} p_{i+1}^{\prime}$ with vertices $p_{i}^{\prime}=\left(x_{i}^{\prime}, b_{1}\right), p_{i+1}^{\prime}=\left(x_{i+1}^{\prime}, b_{1}\right)$.

(2) $q_{i}^{\prime} \in q_{+}^{\prime}$ and $x_{i+1}^{\prime}<x_{i}^{\prime}$. In this case, replace the unit $q_{i}^{\prime} q_{i+1}^{\prime}$ by a new unit $p_{i}^{\prime} p_{i+1}^{\prime}$ with vertices $p_{i}^{\prime}=\left(x_{i}^{\prime}, b_{2}\right), p_{i+1}^{\prime}=\left(x_{i+1}^{\prime}, b_{2}\right)$ if $x_{i}^{\prime}=a_{3}$ and with vertices $p_{i}^{\prime}=\left(x_{i}^{\prime}, b_{3}\right)$, $p_{i+1}^{\prime}=\left(x_{i+1}^{\prime}, b_{3}\right)$ if $x_{i}^{\prime}=a_{2}$.

(3) $q_{i}^{\prime} \in q_{-}^{\prime}$ and $x_{i+1}^{\prime}<x_{i}^{\prime}$. In this case, replace $q_{i}^{\prime} q_{i+1}^{\prime}$ by a new unit $p_{i}^{\prime} p_{i+1}^{\prime}$ with vertices $p_{i}^{\prime}=\left(x_{i}^{\prime}, b_{1}\right), p_{i+1}^{\prime}=\left(x_{i+1}^{\prime}, b_{1}\right)$.

(4) $q_{i}^{\prime} \in q_{-}^{\prime}$ and $x_{i+1}^{\prime}>x_{i}^{\prime}$. In this case, replace $q_{i}^{\prime} q_{i+1}^{\prime}$ by a new unit $p_{i}^{\prime} p_{i+1}^{\prime}$ with vertices $p_{i}^{\prime}=\left(x_{i}^{\prime}, b_{2}\right), p_{i+1}^{\prime}=\left(x_{i+1}^{\prime}, b_{2}\right)$ if $x_{i+1}^{\prime}=a_{3}$ and with vertices $p_{i}^{\prime}=\left(x_{i}^{\prime}, b_{3}\right), p_{i+1}^{\prime}=$ $\left(x_{i+1}^{\prime}, b_{3}\right)$ if $x_{i+1}^{\prime}=a_{2}$.

It is easy to see that after Step 2 the bolt $q^{\prime}$ is replaced by the bolt $p^{\prime}=\left\{p_{1}^{\prime}, p_{2}^{\prime}, \ldots, p_{2 m}^{\prime}\right\}$ and

$$
l\left(f, q^{\prime}\right) \leq l\left(f, p^{\prime}\right)
$$


From (2.10) and (2.11), we deduce that

$$
l(f, p) \leq l\left(f, p^{\prime}\right)
$$

It is clear that each point of the set $p_{+}^{\prime}$ coincides with one of the points $\left(a_{1}, b_{1}\right),\left(a_{2}, b_{3}\right)$, $\left(a_{3}, b_{2}\right)$ and each point of the set $p_{-}^{\prime}$ coincides with one of the points $\left(a_{1}, b_{2}\right),\left(a_{1}, b_{3}\right)$, $\left(a_{2}, b_{1}\right),\left(a_{2}, b_{2}\right),\left(a_{3}, b_{1}\right)$. Denote by $m_{i j}$ the number of points of the bolt $p^{\prime}$ coinciding with the point $\left(a_{i}, b_{j}\right), i, j=\overline{1,3}, i+j \neq 6$. By (2.6), we can write

$$
l\left(f, p^{\prime}\right)=\frac{1}{2 m} \sum_{\substack{i, j=\overline{1,3} \\ i+j \leq 5}} m_{i j} f_{i j} .
$$

On the straight line $x=a_{i}$ or $y=b_{i}, i=\overline{1,3}$, the number of points of the set $p_{+}^{\prime}$ is equal to the number of points of the set $p_{-}^{\prime}$. Hence,

$$
m_{11}=m_{12}+m_{13}=m_{21}+m_{31}, \quad m_{23}=m_{22}+m_{21}=m_{13}, \quad m_{32}=m_{31}=m_{12}+m_{22} .
$$

From these equalities, we deduce that

$$
\begin{gathered}
m_{11}=m_{12}+m_{21}+m_{22}, \quad m_{13}=m_{21}+m_{22}, \\
m_{23}=m_{21}+m_{22}, \quad m_{31}=m_{12}+m_{22} .
\end{gathered}
$$

Consequently,

$$
2 m=\sum_{\substack{i, j=\overline{1,3} \\ i+j \leq 5}} m_{i j}=4 m_{12}+4 m_{21}+6 m_{22}
$$

Considering (2.15) and (2.16) in (2.13) and taking (2.7) into account, we obtain

$$
\begin{aligned}
l\left(f, p^{\prime}\right) & =\frac{4 m_{12}\left|l\left(f, r_{2}\right)\right|+4 m_{21}\left|l\left(f, r_{1}\right)\right|+6 m_{22}|l(f, h)|}{4 m_{12}+4 m_{21}+6 m_{22}} \\
& \leq \max \left\{\left|l\left(f, r_{1}\right)\right|,\left|l\left(f, r_{2}\right)\right|,|l(f, h)|\right\} .
\end{aligned}
$$

Therefore, due to (2.12),

$$
l(f, p) \leq \max \left\{\left|l\left(f, r_{1}\right)\right|,\left|l\left(f, r_{2}\right)\right|,|l(f, h)|\right\} .
$$

Note that in the beginning of the proof the bolt $p$ has been chosen so that $l(f, p) \geq 0$. Let now $p=\left\{p_{1}, p_{2}, \ldots, p_{2 n}\right\}$ be any closed bolt such that $l(f, p) \leq 0$. Since $l\left(f, p^{\prime \prime}\right)=$ $-l(f, p) \geq 0$ for the bolt $p^{\prime \prime}=\left\{p_{2}, p_{3}, \ldots, p_{2 n}, p_{1}\right\}$, we obtain from (2.18) that

$$
-l(f, p) \leq \max \left\{\left|l\left(f, r_{1}\right)\right|,\left|l\left(f, r_{2}\right)\right|,|l(f, h)|\right\} .
$$


From (2.18) and (2.19), we deduce on the strength of arbitrariness of $p$ that

$$
\sup _{p \subset H}\{|l(f, p)|\}=\max \left\{\left|l\left(f, r_{1}\right)\right|,\left|l\left(f, r_{2}\right)\right|,|l(f, h)|\right\},
$$

where the sup is taken over all closed bolts of the hexagon $H$.

The hexagon $H$ satisfies all the conditions of [8, Theorem 3] on the existence of a best approximation. By [9, Theorem 2], we obtain

$$
E(f, H)=\sup _{p \subset H}\{|l(f, p)|\} .
$$

From (2.20) and (2.21), we finally conclude that

$$
E(f, H)=\max \left\{\left|l\left(f, r_{1}\right)\right|,\left|l\left(f, r_{2}\right)\right|,|l(f, h)|\right\} .
$$

Corollary 2.2. Let a function $f(x, y)$ have the continuous nonnegative derivative $\partial^{2} f /$ $\partial x \partial y$ on $H$. Then the formula (2.4) is valid.

The proof is very simple and can be obtained by integrating the inequality $\partial^{2} f / \partial x \partial y \geq$ 0 over an arbitrary rectangle $\left[x_{1}, x_{2}\right] \times\left[y_{1}, y_{2}\right] \subset H$ and applying the above-proven theorem.

Remark 2.3. As seen from the proof of Theorem 2.1, we considerably use the powerful apparatus - bolts of lightning and results closely connected with this notion. Direct generalization of these results (and therefore Theorem 2.1) from our case to the case of approximation of a function $f\left(x_{1}, \ldots, x_{n}\right)$ by sums $\sum_{i=1}^{n} \varphi_{i}\left(x_{i}\right)$ is quite difficult on the simple ground that there is not yet any suitable definition of a bolt of lightning in $n$-dimensional case. All attempts made to solve this problem were not complete and contained gaps in the proof (see $[7,11,12])$. It should be noted that this interesting problem is still open.

\section{E-bolts}

The main idea in the proof of Theorem 2.1 - the closed bolts maximization principlecan be successfully used in obtaining formulas of type (2.4) for functions $f(x, y)$ defined on another simple polygons. The following two theorems include the cases of some octagons and can be proved in a similar way.

Theorem 3.1. Let $a_{1}<a_{2}<a_{3}<a_{4}, b_{1}<b_{2}<b_{3}$ and let $Q$ be an octagon of the following form:

$$
Q=\bigcup_{i=1}^{4} R_{i},
$$

where $R_{1}=\left[a_{1}, a_{2}\right] \times\left[b_{1}, b_{2}\right], R_{2}=\left[a_{2}, a_{3}\right] \times\left[b_{1}, b_{2}\right], R_{3}=\left[a_{3}, a_{4}\right] \times\left[b_{1}, b_{2}\right], R_{4}=\left[a_{2}, a_{3}\right]$ $\times\left[b_{2}, b_{3}\right]$.

Let $f \in M(Q)$. Then

$$
E(f, Q)=\max \left\{|l(f, q)|,\left|l\left(f, r_{123}\right)\right|,\left|l\left(f, r_{124}\right)\right|,\left|l\left(f, r_{234}\right)\right|,\left|l\left(f, r_{24}\right)\right|\right\},
$$


where $q, r_{123}, r_{124}, r_{234}, r_{24}$ are closed bolts formed by the vertices of polygons $Q, R_{1} \cup R_{2} \cup R_{3}$, $R_{1} \cup R_{2} \cup R_{4}, R_{2} \cup R_{3} \cup R_{4}$, and $R_{2} \cup R_{4}$, respectively.

Theorem 3.2. Let $a_{1}<a_{2}<a_{3}<a_{4}, b_{1}<b_{2}<b_{3}$ and let $Q$ be an octagon of the following form:

$$
Q=\bigcup_{i=1}^{3} R_{i},
$$

where $R_{1}=\left[a_{1}, a_{4}\right] \times\left[b_{1}, b_{2}\right], R_{2}=\left[a_{1}, a_{2}\right] \times\left[b_{2}, b_{3}\right], R_{3}=\left[a_{3}, a_{4}\right] \times\left[b_{2}, b_{3}\right]$.

Let $f \in M(Q)$. Then

$$
E(f, Q)=\max \left\{|l(f, r)|,\left|l\left(f, r_{12}\right)\right|,\left|l\left(f, r_{13}\right)\right|\right\},
$$

where $r, r_{12}, r_{13}$ are closed bolts formed by the vertices of polygons $R=\left[a_{1}, a_{4}\right] \times\left[b_{1}, b_{3}\right]$, $R_{1} \cup R_{2}, R_{1} \cup R_{3}$, respectively.

Although the closed bolts maximization process can be applied to bolts of an arbitrary polygon, some combinatorial difficulties arise when grouping values at points of a maximized bolt (a bolt obtained after maximization process) as we have done it in the proof of Theorem 2.1 (see (2.13)-(2.18)). While we cannot give a complete answer to this problem, we can describe the points of a polygon $F$ with which points of maximized bolts coincide and state a conjecture concerning the approximation error.

Let $F=A_{1} A_{2} \cdots A_{2 n}$ be a polygon with sides, as mentioned in the introduction, parallel to coordinate axes. The vertices $A_{1}, A_{2}, \ldots, A_{2 n}$ in the given order form a closed bolt of lightning, which we denote by $r_{F}$. The length $\left[r_{F}\right]$ of the bolt $r_{F}$ is a number of points forming $r_{F}$. In our case, $\left[r_{F}\right]=2 n$.

Definition 3.3. Let $F$ and $S$ be polygons with sides parallel to coordinate axes. The closed bolt $r_{F}$ is an $e$-bolt (extended bolt) of $S$ if $r_{F} \subset S$ and there does not exist any polygon $F^{\prime}$ such that $F \subset F^{\prime}, r_{F^{\prime}} \subset S,\left[r_{F^{\prime}}\right] \leq\left[r_{F}\right]$.

For example, in Theorem 3.2, the octagon $Q$ has $3 e$-bolts. They are $r, r_{12}$, and $r_{13}$. In Theorem 3.1, the octagon $Q$ has $5 e$-bolts, which are $q, r_{123}, r_{124}, r_{234}$, and $r_{24}$. The polygon $S_{2 n}=\bigcup_{i=1}^{n-1} R_{i}$, where $R_{i}=\left[a_{i}, a_{i+1}\right] \times\left[b_{1}, b_{n+1-i}\right], i=\overline{1, n-1}, a_{1}<a_{2}<\cdots<a_{n}$, $b_{1}<b_{2}<\cdots<b_{n}$, has exactly $2^{n-1}-1 e$-bolts. It is not difficult to observe that the set of points of a closed bolt obtained after maximization process is a subset of the set of points of all $e$-bolts. This condition and Theorems 2.1, 3.1, and 3.2 justify the statement of the following conjecture.

Conjecture 3.4. Let $S$ be any polygon with sides parallel to coordinate axes and $f(x, y) \in$ $M(S)$. Then

$$
E(f, S)=\max _{h \in S^{B}}\{|l(f, h)|\}
$$

where $S^{B}$ is a set of all $e$-bolts of the polygon $S$. 


\section{Error estimates}

Theorem 2.1 enables us to consider classes wider than $M(H)$ and establish sharp estimates for the approximation error.

Theorem 4.1. Let $H$ be a hexagon and let (2.1) be its representation. The following sharp estimates are valid for a function $f(x, y)$ having the continuous derivative $\partial^{2} f / \partial x \partial y$ on $H$ :

$$
A \leq E(f, H) \leq B C+\frac{3}{2}(B|l(g, h)|-|l(f, h)|),
$$

where

$$
\begin{gathered}
B=\max _{(x, y) \in H}\left|\frac{\partial^{2} f(x, y)}{\partial x \partial y}\right|, \quad g=g(x, y)=x \cdot y, \\
A=\max \left\{|l(f, h)|,\left|l\left(f, r_{1}\right)\right|,\left|l\left(f, r_{2}\right)\right|\right\}, \quad C=\max \left\{|l(g, h)|,\left|l\left(g, r_{1}\right)\right|,\left|l\left(g, r_{2}\right)\right|\right\},
\end{gathered}
$$

$h, r_{1}, r_{2}$ are closed bolts formed by vertices of the polygons $H, R_{1}$, and $R_{2}$, respectively.

Remark 4.2. Equation (4.1)-type inequalities were established in [2] for the approximation of a function $f(x)=f\left(x_{1}, \ldots, x_{n}\right)$, defined on a parallelepiped with sides parallel to coordinate axes, by sums $\sum_{i=1}^{n} \varphi_{i}\left(x \backslash x_{i}\right)$. For the approximation of bivariate functions, Babaev's result contains only rectangular case. On the other hand, this type of approximation is not a generalization of the type of approximation considered here, as functions $\varphi_{i}\left(x \backslash x_{i}\right)=\varphi_{i}\left(x_{1}, \ldots, x_{i-1}, x_{i+1}, \ldots, x_{n}\right)$ are not univariate (see Remark 2.3).

Remark 4.3. Estimates (4.1) are easily calculable in contrast to those established in [3] for any continuous function defined on certain domains, which are different from a polygon with the number of sides more than 4 .

To prove Theorem 4.1, we need the following lemmas.

Lemma 4.4. Let $X$ be a normed space and let $F$ be a subspace of $X$. The following inequalities are valid for any element $x=x_{1}+x_{2}$ from $X$ :

$$
\left|E\left(x_{1}\right)-E\left(x_{2}\right)\right| \leq E(x) \leq E\left(x_{1}\right)+E\left(x_{2}\right),
$$

where

$$
E(x)=E(x, F)=\inf _{y \in F}\|x-y\| .
$$

Lemma 4.5. If $f \in M(H)$, then

$$
\left|l\left(f, r_{i}\right)\right| \leq \frac{3}{2}|l(f, h)|, \quad i=1,2 .
$$

Lemma 4.4 is obvious. To prove Lemma 4.5 , note that for any $f \in M(H)$,

$$
6|l(f, h)|=4\left|l\left(f, r_{i}\right)\right|+4\left|l\left(f, r_{3}\right)\right|, \quad i=1,2,
$$

where $r_{3}$ is a closed bolt formed by the vertices of the rectangle $R_{3}=H \backslash R_{i}$. 
Proof. It is not difficult to verify that if $\partial^{2} u / \partial x \partial y \geq 0$ on $H$ for some $u(x, y), \partial^{2} u(x, y) / \partial x \partial y$ $\in C(H)$, then $u \in M(H)$ (see the proof of Corollary 2.2). Set $f_{1}=f+B g$. Since $\partial^{2} f_{1} / \partial x \partial y \geq 0$ on $H, f_{1} \in M(H)$. By Lemma 4.5 ,

$$
\left|l\left(f_{1}, r_{i}\right)\right| \leq \frac{3}{2}\left|l\left(f_{1}, h\right)\right|, \quad i=1,2 .
$$

Theorem 2.1 implies that

$$
E\left(f_{1}, H\right)=\max \left\{\left|l\left(f_{1}, h\right)\right|,\left|l\left(f_{1}, r_{1}\right)\right|,\left|l\left(f_{1}, r_{2}\right)\right|\right\} .
$$

We deduce from (4.7) and (4.8) that

$$
E\left(f_{1}, H\right) \leq \frac{3}{2}\left|l\left(f_{1}, h\right)\right|
$$

First, let the closed bolt $h$ start at the point $\left(a_{1}, b_{1}\right)$. Then it is clear that

$$
E\left(f_{1}, H\right) \leq \frac{3}{2} l\left(f_{1}, h\right)
$$

By Lemma 4.4,

$$
E(f, H)-E(B g, H) \leq E\left(f_{1}, H\right) .
$$

Inequalities (4.10) and (4.11) yield

$$
E(f, H) \leq B E(g, H)+\frac{3}{2} l\left(f_{1}, h\right) .
$$

Since the functional $l(f, h)$ is linear,

$$
l\left(f_{1}, h\right)=l(f, h)+B l(g, h)
$$

Considering this expression of $l\left(f_{1}, h\right)$ in $(4.12)$, we get

$$
E(f, H) \leq B E(g, H)+\frac{3}{2} B l(g, h)+\frac{3}{2} l(f, h) .
$$

Now set $f_{2}=B g-f$. Then $\partial^{2} f_{2} / \partial x \partial y \geq 0$ on $H$. It can be shown, by the same way as (4.14) has been obtained, that

$$
E(f, H) \leq B E(g, H)+\frac{3}{2} B l(g, h)-\frac{3}{2} l(f, h) .
$$


From (4.14) and (4.15), it follows that

$$
E(f, H) \leq B E(g, H)+\frac{3}{2} B l(g, h)-\frac{3}{2}|l(f, h)| .
$$

Note that since $g \in M(H)$ and $h$ starts at the point $\left(a_{1}, b_{1}\right), l(g, h) \geq 0$.

Let now $h$ start at a point such that $l(u, h) \leq 0$ for any $u \in M(H)$, hence for $g, f_{1}$, and $f_{2}$. Then applying the same techniques from the beginning of the proof up to (4.16), we obtain

$$
E(f, H) \leq B E(g, H)-\frac{3}{2} B l(g, h)-\frac{3}{2}|l(f, h)|,
$$

where $l(g, h) \leq 0$. From (4.16), (4.17) and the fact that $E(g, H)=C$ (in view of Theorem $2.1)$, it follows that

$$
E(f, H) \leq B C+\frac{3}{2}(B|l(g, h)|-|l(f, h)|) .
$$

The upper bound in (4.1) has been established. Notice that it is attained by $f=g=x y$.

Proof of the lower bound in (4.1) is simple. One of the obvious properties of the functional $l(f, p)$ is that $|l(f, p)| \leq E(f, H)$ for any continuous function $f$ on $H$ and a closed bolt $p$. Hence,

$$
A=\max \left\{|l(f, h)|,\left|l\left(f, r_{1}\right)\right|,\left|l\left(f, r_{2}\right)\right|\right\} \leq E(f, H) .
$$

Notice that by Theorem 2.1 the lower bound in (4.1) is attained by any function from $M(H)$.

Remark 4.6. In view of Theorems 3.1 and 3.2 and the ideas set forth in Section 3, it is possible to establish sharp estimates of type (4.1) for bivariate functions defined on another simple polygons with sides parallel to coordinate axes.

\section{Acknowledgment}

The author thanks the referee for many helpful comments that led to an improvement of the original manuscript.

\section{References}

[1] V. I. Arnol'd, On functions of three variables, Doklady Akademii Nauk SSSR 114 (1957), 679-681, English translation in American Mathematical Society Translations 28 (1963), 51-54.

[2] M.-B. A. Babaev, Sharp estimates for the approximation of functions of several variables by sums of functions of a lesser number of variables, Matematicheskie Zametki 12 (1972), 105-114.

[3] M.-B. A. Babaev and V. E. Ismailov, Two-sided estimates for the best approximation in domains different from the parallelepiped, Functiones et Approximatio Commentarii Mathematici 25 (1997), $121-128$.

[4] R. E. Bank, An automatic scaling procedure for a D'Yakanov-Gunn iteration scheme, Linear Algebra and Its Applications 28 (1979), 17-33.

[5] R. C. Buck, On approximation theory and functional equations, Journal of Approximation Theory 5 (1972), 228-237. 
[6] S. P. Diliberto and E. G. Straus, On the approximation of a function of several variables by the sum of functions of fewer variables, Pacific Journal of Mathematics 1 (1951), 195-210.

[7] N. Dyn, A straightforward generalization of Diliberto and Straus' algorithm does not work, Journal of Approximation Theory 30 (1980), no. 4, 247-250.

[8] A. L. Garkavi, V. A. Medvedev, and S. Ya. Khavinson, On the existence of a best uniform approximation of a function in two variables by sums $\varphi(x)+\psi(y)$, Sibirskil Matematicheskiu Zhurnal 36 (1995), no. 4, 819-827, English translation in Siberian Mathematical Journal 36 (1995), no. 4, 707-713.

[9] S. Ja. Havinson, A Čebyšsv theorem for the approximation of a function of two variables by sums $\varphi(x)+\psi(y)$, Izvestiya Akademii Nauk SSSR. Seriya Matematicheskaya 33 (1969), 650-666, English translation in Mathematics of the USSR-Izvestiya 3 (1969), 617-632.

[10] V. E. Ismailov, On some classes of bivariate functions characterized by formulas for the best approximation, Radovi Matematički 13 (2004), no. 1, 53-62.

[11] D. E. Marshall and A. G. O'Farrell, Approximation by a sum of two algebras. The lightning bolt principle, Journal of Functional Analysis 52 (1983), no. 3, 353-368.

[12] V. A. Medvedev, Refutation of a theorem of Diliberto and Straus, Matematicheskie Zametki 51 (1992), no. 4, 78-80, English translation in Mathematical Notes 51, (1992), no. 3-4, 380-381.

[13] Ju. P. Ofman, On the best approximation of functions of two variables by functions of the form $\varphi(x)+\psi(y)$, Izvestiya Akademii Nauk SSSR. Seriya Matematicheskaya 25 (1961), 239-252, English translation in American Mathematical Society Translations 44 (1965), 12-28.

[14] T. J. Rivlin and R. J. Sibner, The degree of approximation of certain functions of two variables by a sum of functions of one variable, The American Mathematical Monthly 72 (1965), 1101-1103.

[15] Y. Sternfeld, Uniformly separating families of functions, Israel Journal of Mathematics 29 (1978), no. 1, 61-91.

Vugar E. Ismailov: Institute of Mathematics and Mechanics, Azerbaijan National Academy of Sciences, 9 F. Agaev Street, 1141 Baku, Azerbaijan

E-mail address: vugaris@mail.ru 


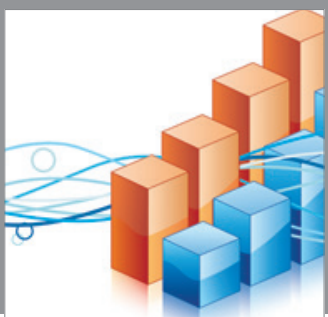

Advances in

Operations Research

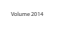

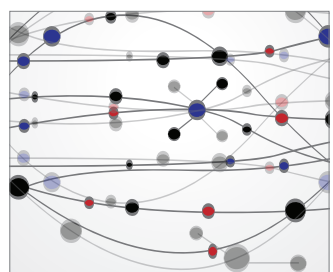

\section{The Scientific} World Journal
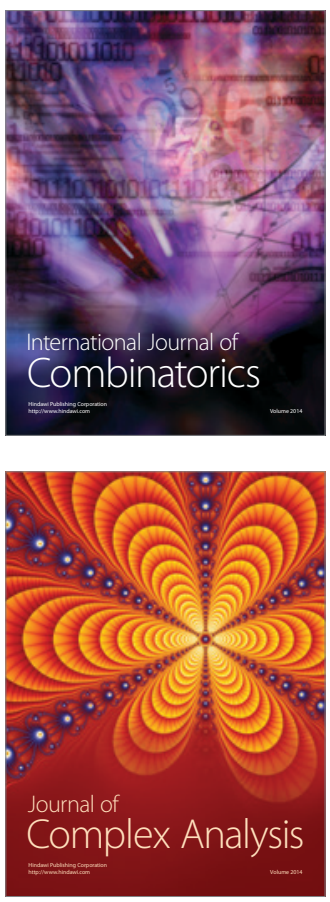

International Journal of

Mathematics and

Mathematical

Sciences
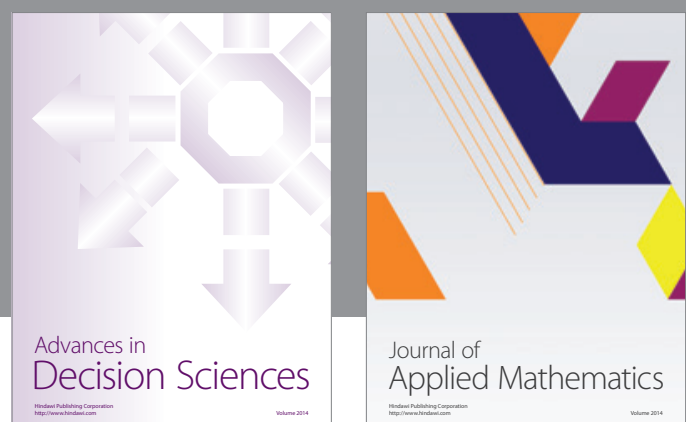

Journal of

Applied Mathematics
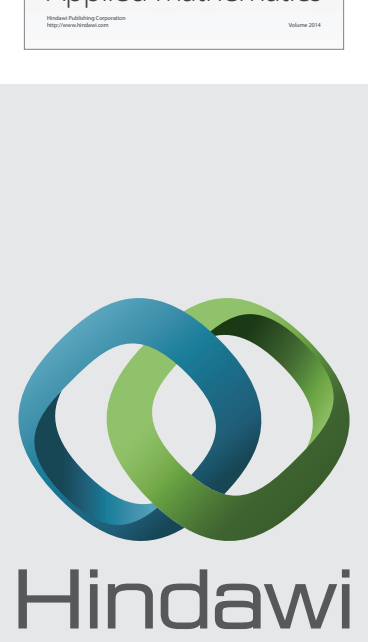

Submit your manuscripts at http://www.hindawi.com
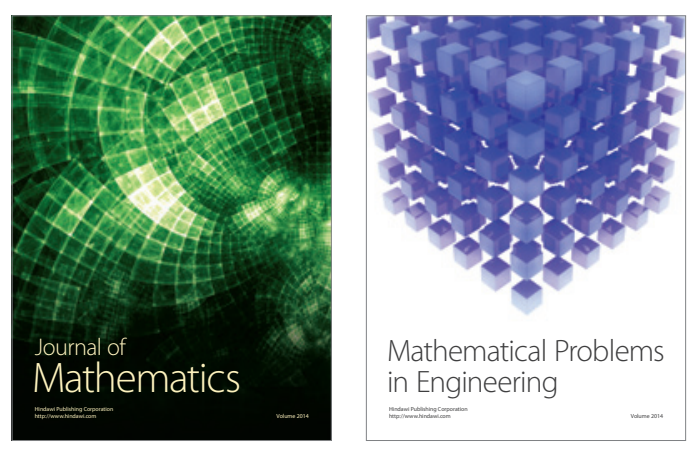

Mathematical Problems in Engineering
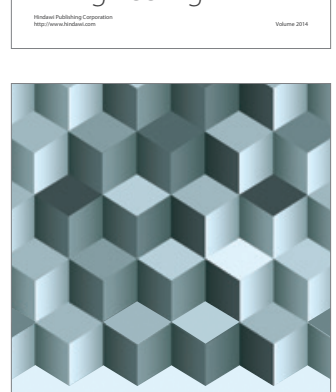

Journal of

Function Spaces
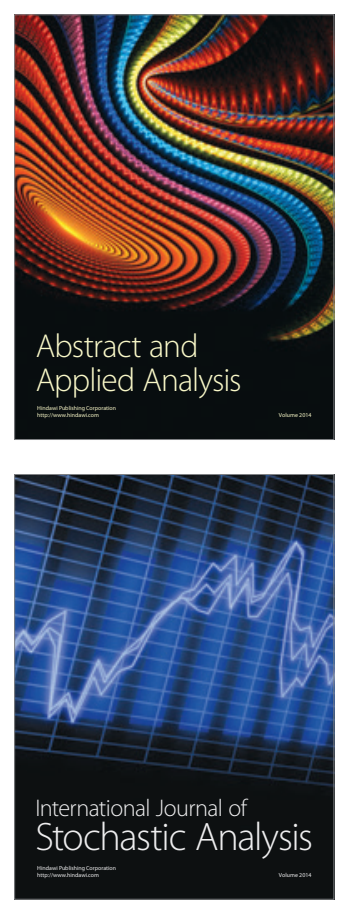

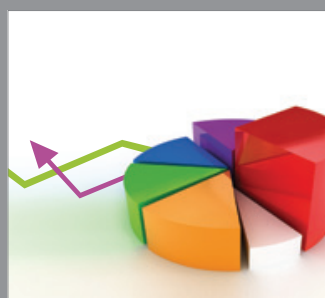

ournal of

Probability and Statistics

Promensencen
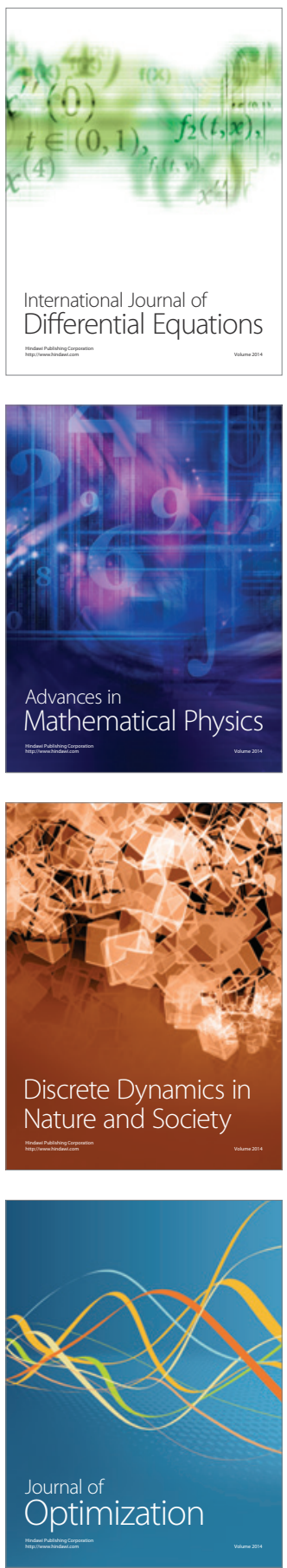